Research Paper

\title{
Ultrasound for Measuring the Cross-Sectional Area of Biceps Brachii Muscle in Sarcopenia
}

\author{
Shumin $\mathrm{Li}^{1}$, Hanyu Li1 ${ }^{1}$, Ying $\mathrm{Hu}^{2}$, Shaoming Zhu ${ }^{2}$, Zherong $\mathrm{Xu}^{1}$, Qin Zhang ${ }^{1,3}$, Yunmei Yang ${ }^{1,3}{ }^{凶}$, Zhaodi
} Wang 1,3 , Jia $\mathrm{Xu}^{3,4}$

1. Department of Geriatrics, the First Affiliated Hospital, School of Medicine, Zhejiang University, Hangzhou, 310003, China

2. Department of Ultrasound, The First Affiliated Hospital, School of Medicine, Zhejiang University, Hangzhou, 310003, China

3. Zhejiang Provincial Key Laboratory for Diagnosis and Treatment of Aging and Physic-chemical Injury Diseases, the First Affiliated Hospital, School of Medicine, Zhejiang University, Hangzhou, 310003, China

4. Department of Emergency Medicine, The First Affiliated Hospital, School of Medicine, Zhejiang University, Hangzhou, 310003, China

$\square$ Corresponding author: Yunmei Yang, Department of Geriatrics, The First Affiliated Hospital, School of Medicine, Zhejiang University, Hangzhou, Zhejiang, China. Tel: +86-571-87236808; Email: 1194070@zju.edu.cn

(1) The author(s). This is an open access article distributed under the terms of the Creative Commons Attribution License (https://creativecommons.org/licenses/by/4.0/). See http:/ /ivyspring.com/terms for full terms and conditions.

Received: 2020.06.18; Accepted: 2020.09.27; Published: 2020.10.18

\begin{abstract}
Background: Ultrasound is emerging as an effective method for measuring muscle mass in elderly people. It has been applied in numerous studies to obtain measurement of lower limbs. The study aims to explore the relationship between sarcopenia and ultrasound measurements of biceps brachii.

Methods: Participants $(n=179)$ aged over 60 years were enrolled from the first affiliated hospital of Zhejiang University. The muscle thickness (MT), cross-sectional area (CSA) and fat thickness (FT) of these participants were recorded. Spearman test and partial correlation test was used to determine the correlation between indicators. Mann-Whitney $U$ test was performed to compare ultrasonic parameters between sarcopenia group and non-sarcopenia group. The binary logistic regression analysis was employed to detect the potential indicators and prediction equation of sarcopenia. Receiver operating characteristic (ROC) curve analysis was performed for the accuracy of equation.

Results: The prevalence of sarcopenia were $16.3 \%$ and $10.8 \%$ respectively in men and women. CSA was significantly lower in sarcopenia group than non-sarcopenia group in women $(P<0.05)$. CSA was positively correlated with skeletal muscle mass index (SMI) and grip strength (men: $r=0.460,0.433$; women: $r=0.267,0.392$ ). After controlling of age and BMI, these correlations disappeared. Binary logistic regression analysis showed that age $(O R=1.149,95 \% \mathrm{Cl}: 1.060-1.246 ; P=0.001)$ and $C S A(O R=0.465$, 95\% Cl: 0.225-0.963; $P=0.039)$ was significant indicators associated with sarcopenia. Area Under Curve was $0.822(95 \% \mathrm{Cl}: 0.725-0.919, \mathrm{P}<0.001)$ for the prediction equation composed of age, gender and CSA for sarcopenia.

Conclusion: CSA of the biceps brachii measured with ultrasound is an important indicator associated with sarcopenia.
\end{abstract}

Key words: sarcopenia, ultrasound, biceps brachii, muscle mass

\section{Introduction}

Sarcopenia, first proposed by Irwin Rosenberg in 1989 , is characterized by a progressive loss of skeletal muscle mass and is associated with aging[1]. It decreases muscle strength and function. It was until 2016 when the Centers for Disease Control and Prevention approved the international classification of sarcopenia as an independent disease[2]. Sarcopenia severely deteriorates the health of elderly people and increases the risks of falls, fractures, disability, and mortality, especially in those with chronic diseases[3]. The diagnostic criteria for sarcopenia are not yet uniform. The diagnose index of sarcopenia includes the skeletal muscle mass, muscle strength (usually reflected by grip strength) and physical performance (usually reflected by gait speed)[4-7]. Because skeletal muscle mass is 
considered as one of the important indicators for sarcopenia, its measurement methods have received widespread attention. Dual-energy X-ray absorptiometry (DXA) and bio-electrical impedance analysis (BIA) are widely used to assess skeletal muscle mass. Computed tomography (CT) and magnetic resonance imaging (MRI) have been recommended as the gold standard for assessing muscle mass because they can clearly show the tissue structure. Ultrasound, being convenience and pocket-friendly, is increasingly being valued in the evaluation of skeletal muscle mass.

Although the literature and guideline suggest that the gold standard for muscle mass testing is CT or MRI[8, 9], these two measurements have some limitations. They are time-consuming, expensive and require specialized equipment. The $\mathrm{CT}$ can even cause radiation to people. Due to these limitations, the use of CT and MRI evaluating muscle mass in the elderly has not yet peaked. Although the cut-off points of CT for sarcopenia had been reported, the practical application is still limited[10]. Although DXA has been widely accepted as the measurement of skeletal muscle mass, it has some limitations, such as radiation, cannot operate at bedside and deficiency in primary hospital. The BIA is a simple and convenient technology that is easily accepted and can operate at the bedside. Its reliability is however affected by various factors, such as an electrode, operator, environment and different machines[11]. In addition, the assessment results of DXA and BIA are different which causes nonuniform standard for sarcopenia to diagnose[12]. Therefore, there is an urgent need for a standard and uniform measurement of sarcopenia diagnose.

Ultrasound is widely used to diagnose and follow-up in the clinic. It can distinguish muscle tissue from subcutaneous fat and show the thickness and cross-sectional area of muscle. Ultrasound is a convenient, reliable and non-radiative technique, and can be performed at the bedside for those who cannot cooperate with DXA, CT or MRI. Compared with the above methods, the application of ultrasound is more common, and many people are more willing to accept the ultrasound examination, which has a great prospect in the application for sarcopenia. It has been pointed out as an effective method to assess muscle mass in the elderly[13]. Good consistency between the thickness of the thigh muscle measured by ultrasound and the muscle mass by DXA has been shown[14].

The commonly used indices for assessing muscle mass by ultrasound include muscle thickness (or diameter), muscle cross-sectional area, muscle fascicle length, pinnation angle and muscle echo[15]. Most of the current studies on the evaluation of sarcopenia by ultrasound have focused on lower limb ultrasound, especially the quadriceps femoris and gastrocnemius muscles[15, 16]. Few studies have focused on the importance of assessing upper limbs using ultrasound. For a better understanding of the biceps muscle thickness (MT), cross-sectional area (CSA) and fat thickness (FT) measured by ultrasound related to sarcopenia, this study analyzed the relationship among FT, MT and CSA of biceps and muscle mass, grip strength and sarcopenia. The aim of the study is to provide a theoretical basis for the application of ultrasound in sarcopenia in the future.

\section{Methods}

\section{Participants}

All participants were from the health management center of the first affiliated hospital of Zhejiang University medical college. History and clinical examination of all participants were assessed. All the participants were more than 60 years old and willing to accept the examinations of DXA and ultrasound. The elderly with restricted activities such as limb hemiplegia or limb fracture within three months, muscle-related diseases such as myositis, progressive muscular dystrophy, and myasthenia gravis; dementias and those who could not cooperate with the test were excluded. A total of 179 participants were sampled for the study. The study was approved by the ethics committee and informed consent obtained from all the participants.

\section{Ultrasound measurements}

The biceps FT, MT, and CSA of all participants were measured by the same examiner[17]. The participants were in the supine position with their limbs extended and relaxed. The examiner held the ultrasound probe $(2-10 \mathrm{MHz}$, Aixplorer; Aix-en-Provence, France) vertically against the skin surface to measure accurately to $0.01 \mathrm{~cm}$ the maximum cross-sectional area of the biceps brachii muscle in the dominant hand. It was used as a marker to detect its axial view of Fat thickness and muscle thickness. The measurement was repeated after a 10-minute rest. The average measurement was calculated and used in the analysis.

\section{Muscle mass}

The muscle mass of participants was assessed by DXA (DISCOVERY-W, Hologic, Bedford, MA, USA). The participants were requested to take off their clothes and the measurements were taken without metal to eliminate artifacts that could be caused by the clothes. Body composition was assessed by scanning the body. Skeletal muscle mass index (SMI) was analyzed and recorded using height-adjusted muscle 
mass (limb muscle mass / height ${ }^{2}$ ) accurate to 0.1 $\mathrm{kg} / \mathrm{m}$ squared.

\section{Grip strength}

JAMAR manual dynamometer (Asimow Engineering, Los Angeles, CA, USA) was used to measure grip strength as per the procedures by Roberts et al[18]. The participant sat comfortably on a standard chair (with backrest and flat armrests) with their forearms resting on the armrests. The participant was requested to squeeze the JAMAR until the pointer stopped rising. Measurements were taken alternately with each hand, three times on each side. The highest result was recorded, accurate to $0.1 \mathrm{~kg}$.

\section{Gait speed}

A 4-meter walking test was performed to measure gait speed [19]. Participants were asked to stand still behind a marked line. They started walking at their normal pace and stopped near the finish line. The maximum value of the two measurements was taken for evaluation, and the recorded value was accurate to $0.1 \mathrm{~m} / \mathrm{s}$.

\section{Group Definition}

The cut-off point recommended by the Asian sarcopenia working group was used as the diagnostic criteria [4]. The SMI less than $7.0 \mathrm{~kg} / \mathrm{m}^{2}$ in men and less than $5.4 \mathrm{~kg} / \mathrm{m}^{2}$ in women belonged to the low muscle mass. The grip strength below $26 \mathrm{~kg}$ and $18 \mathrm{~kg}$ in men and women respectively belonged to the low grip strength. Low muscle mass, along with low grip strength or low gait speed $(<0.8 \mathrm{~m} / \mathrm{s}$, both men and women), belonged to the sarcopenia group, and the rest belonged to the non-sarcopenia group.

\section{Statistical analyses}

SPSS 24.0 statistical software was employed to analyze the data. The Shapiro-Wilk test was performed to test the normality. Since most of the data did not conform to the normal distribution, the Mann-Whitney $U$ test was performed to compare two groups. The experimental data were shown as median and quartile (M(P25, P75)). Chi-square test is used for categorical data. Spearman test was used for correlation analysis. Partial correlation test was used for analysis after controlling of age and BMI. Binary logistic regression analysis was used to detect the potential indicators and prediction equation for sarcopenia. Based on the result of binary logistic regression and formula of logistic model: $\operatorname{logit}(P)=$ In $[P /(1-P)]=\beta_{0}+\beta_{1} X_{1}+\ldots+\beta \mathrm{nXn}$ (the value of $\beta$ comes from logistic regression, $X$ is the independent variable, $\mathrm{n}$ is the number of independent variables), we can get the value of probability for dependent variable: $P=\mathrm{e}^{\operatorname{logit}(P)} / 1+\mathrm{e}^{\operatorname{logit}(P)}$. The accuracy of equation is assessed by receiver operating characteristic (ROC) curve analysis. The statistical value of $p<0.05$ was used to test significance.

\section{Results}

A total 179 participants were recruited, 0 was excluded. There were 8 participants in men and 14 participants in women who were diagnosed as sarcopenia. The prevalence of sarcopenia was $16.3 \%$ and $10.8 \%$ respectively in men and women. Men were significantly higher than women in terms of height, weight, BMI, muscle mass, grip strength, MT, and CSA. While FT was significantly lower in men than in women. There was no significant difference in age and gait speed between two groups (Table 1). The baseline information between sarcopenia group and non-sarcopenia group is shown at Supplementary Table 1.

Table 1. Differences between gender

\begin{tabular}{|c|c|c|c|}
\hline & $\operatorname{Men}(n=49)$ & women $(n=130)$ & $\mathrm{P}$ \\
\hline \multicolumn{4}{|l|}{ General information } \\
\hline age(year) & $70(66,80)$ & $69(64,77)$ & 0.180 \\
\hline Height(cm) & $169(166,174)$ & $156(152,160)$ & $<0.001$ \\
\hline Weight(kg) & $70.0(61.5,79.0)$ & $57.8(51.7,62.0)$ & $<0.001$ \\
\hline $\operatorname{BMI}\left(\mathrm{kg} / \mathrm{m}^{2}\right)$ & $24.2(22.4,27.1)$ & $23.4(21.7,25.4)$ & 0.045 \\
\hline \multicolumn{4}{|c|}{ Sarcopenia assessment } \\
\hline $\mathrm{SMI}\left(\mathrm{kg} / \mathrm{m}^{2}\right)$ & $6.3(5.7,7.5)$ & $5.3(4.5,6.3)$ & $<0.001$ \\
\hline Grip strength $(\mathrm{kg})$ & $35.9(32.5,40.3)$ & $22.9(20.0,26.0)$ & $<0.001$ \\
\hline Gait speed(m/s) & $1.2(1.0,1.4)$ & $1.2(1.0,1.3)$ & 0.052 \\
\hline \multicolumn{4}{|c|}{ Ultrasound assessment } \\
\hline $\mathrm{FT}(\mathrm{cm})$ & $0.23(0.16,0.31)$ & $0.33(0.25,0.61)$ & $<0.001$ \\
\hline $\mathrm{MT}(\mathrm{cm})$ & $2.14(2.03,2.48)$ & $1.69(1.47,1.90)$ & $<0.001$ \\
\hline $\operatorname{CSA}\left(\mathrm{cm}^{2}\right)$ & $8.88(7.03,10.36)$ & $5.46(4.50,6.05)$ & $<0.001$ \\
\hline \multicolumn{4}{|c|}{$\begin{array}{l}\text { Notes: Mann-whitey } U \text { test was used for comparison between two groups. The da } \\
\text { were shown as median and quartile }(\mathrm{M}(\mathrm{P} 25, \mathrm{P} 75)) \text {. Bold means } \mathrm{P}<0.05 \text {, bold }+ \\
\text { italics means } \mathrm{P}<0.01 \text {. }\end{array}$} \\
\hline \multicolumn{4}{|c|}{$\begin{array}{l}\text { Abbreviations: BMI, body mass index; SMI, skeletal muscle mass index; FT, fat } \\
\text { thickness; MT, muscle thickness; CSA, cross-sectional area. }\end{array}$} \\
\hline
\end{tabular}

For women, the FT and CSA were significantly higher in the non-sarcopenia group than those in sarcopenia group. No statistically significant differences in ultrasound parameters were found between two groups in men (Table 2).

A negative correlation between age and grip strength, gait speed, MT and CSA was found in both men and women. A positive correlation was observed between CSA and SMI or grip strength. (Table 3) After controlling of age and BMI, correlations between SMI, grip strength, gait speed, and ultrasound parameters are not significant.

Age, gender, BMI, FT, MT, and CSA were included in the binary logistic regression analysis for sarcopenia. It showed that age $(\mathrm{OR}=1.149,95 \% \mathrm{CI}$ : 1.060-1.246; $P=0.001)$ and CSA (OR=0.465, 95\%CI: $0.225-0.963 ; P=0.039)$ were significant indicators associated with sarcopenia (Table 4). 
Table 2. differences between sarcopenia group and non-sarcopenia group

\begin{tabular}{llll}
\hline & Non-sarcopenia group & sarcopenia group & \\
\hline male & $\mathrm{n}=41$ & $\mathrm{n}=8$ & $\mathrm{P}$ \\
age $($ year $)$ & $69(65,77)$ & $79(74,83)$ & $\mathbf{0 . 0 1 3}$ \\
BMI $\left(\mathrm{kg} / \mathrm{m}^{2}\right)$ & $25.1(23.1,27.4)$ & $21.8(19.5,23.7)$ & $\mathbf{0 . 0 0 3}$ \\
SMI $\left(\mathrm{kg} / \mathrm{m}^{2}\right)$ & $6.5(5.8,7.7)$ & $5.5(4.8,5.8)$ & $\mathbf{0 . 0 0 2}$ \\
Grip strength $(\mathrm{kg})$ & $37.1(33.8,42.0)$ & $26.4(17.0,32.5)$ & $<\mathbf{0 . 0 0 1}$ \\
Gait speed(m/s) & $1.3(1.1,1.4)$ & $0.7(0.6,1.1)$ & $\mathbf{0 . 0 0 7}$ \\
FT $(\mathrm{cm})$ & $0.26(0.18,0.33)$ & $0.20(0.14,0.25)$ & 0.386 \\
MT $(\mathrm{cm})$ & $2.20(2.03,2.63)$ & $2.08(1.97,2.11)$ & 0.158 \\
CSA $\left(\mathrm{cm}{ }^{2}\right)$ & $9.24(7.37,10.67)$ & $7.06(6.40,9.00)$ & 0.075 \\
female & $\mathrm{n}=116$ & $\mathrm{n}=14$ & $\mathrm{P}$ \\
age $($ year $)$ & $68(64,76)$ & $80(74,82)$ & $\mathbf{0 . 0 0 1}$ \\
BMI $\left(\mathrm{kg} / \mathrm{m}^{2}\right)$ & $23.4(22.0,25.5)$ & $22.5(20.1,24.6)$ & 0.150 \\
SMI $\left(\mathrm{kg} / \mathrm{m}^{2}\right)$ & $5.4(4.7,6.5)$ & $4.5(4.1,5.0)$ & $\mathbf{0 . 0 0 1}$ \\
Grip strength(kg) & $23.3(20.7,26.3)$ & $16.4(12.6,18.2)$ & $<\mathbf{0 . 0 0 1}$ \\
Gait speed(m/s) & $1.2(1.1,1.3)$ & $0.8(0.6,0.9)$ & $<\mathbf{0 . 0 0 1}$ \\
FT $(\mathrm{cm})$ & $0.35(0.26,0.42)$ & $0.23(0.19,0.31)$ & $\mathbf{0 . 0 0 1}$ \\
MT $(\mathrm{cm})$ & $1.71(1.47,1.92)$ & $1.61(1.33,1.74)$ & 0.080 \\
CSA $\left(\mathrm{cm}{ }^{2}\right)$ & $5.50(4.60,6.12)$ & $4.40(3.34,5.49)$ & $\mathbf{0 . 0 0 3}$ \\
\hline
\end{tabular}

Notes: Mann-whitey U test was used for comparison between two groups. The data were shown as median and quartile $(\mathrm{M}(\mathrm{P} 25, \mathrm{P} 75))$. Bold means $\mathrm{P}<0.05$, bold + italics means $\mathrm{P}<0.01$.

Abbreviations: BMI, body mass index; FT, fat thickness; MT, muscle thickness; CSA, cross-sectional area.

Table 3. The correlation between indicators

\begin{tabular}{|c|c|c|c|c|c|c|c|c|}
\hline correlation & age & BMI & SMI & Grip strength & Gait speed & FT & MT & CSA \\
\hline \multicolumn{9}{|l|}{ male } \\
\hline Age & 1.000 & -0.121 & -0.419 & -0.566 & -0.357 & 0.029 & -0.307 & -0.319 \\
\hline BMI & & 1.000 & 0.479 & 0.337 & 0.121 & 0.628 & 0.431 & 0.501 \\
\hline SMI & & & 1.000 & 0.347 & 0.162 & 0.268 & 0.359 & 0.460 \\
\hline Grip strength & & & & 1.000 & 0.128 & 0.144 & 0.399 & 0.433 \\
\hline Gait speed & & & & & 1.000 & 0.093 & 0.122 & 0.097 \\
\hline FT & & & & & & 1.000 & 0.226 & 0.107 \\
\hline MT & & & & & & & 1.000 & 0.841 \\
\hline CSA & & & & & & & & 1.000 \\
\hline \multicolumn{9}{|l|}{ female } \\
\hline Age & 1.000 & -0.028 & -0.030 & -0.384 & -0.505 & -0.106 & -0.235 & -0.318 \\
\hline BMI & & 1.000 & 0.327 & 0.013 & 0.060 & 0.491 & 0.245 & 0.246 \\
\hline SMI & & & 1.000 & 0.109 & 0.052 & 0.226 & 0.121 & 0.267 \\
\hline Grip strength & & & & 1.000 & 0.459 & 0.145 & 0.317 & 0.392 \\
\hline Gait speed & & & & & 1.000 & 0.080 & 0.159 & 0.214 \\
\hline FT & & & & & & 1.000 & 0.463 & 0.249 \\
\hline MT & & & & & & & 1.000 & 0.749 \\
\hline CSA & & & & & & & & 1.000 \\
\hline
\end{tabular}

Notes: Spearman test was used for correlation analysis. Bold means $\mathrm{P}<0.05$, bold + italics means $\mathrm{P}<0.01$.

Abbreviations: BMI, body mass index; SMI, skeletal muscle mass index; FT, fat thickness; MT, muscle thickness; CSA, cross-sectional area.

Age, gender and CSA were included into the binary logistic regression analysis again to establish the prediction equation for sarcopenia: $\operatorname{logit}(P)=$ $7.542+0.125^{*}$ age $-1.584^{*}$ gender $($ man $=0$, woman $=1)-$ $0.449^{*} \mathrm{CSA}, P=\mathrm{e}^{\operatorname{logit}(P)} / 1+\mathrm{e}^{\operatorname{logit}(P)}$. ROC curves analyses showed that AUC (Area Under Curve) was 0.822 (95\%CI: 0.725-0.919, $P<0.001$ ) (Figure 1). While the AUC was 0.802 (95\%CI: 0.688-0.916, $\mathrm{P}<0.001)$ when the equation consists of BMI, FT, MT and CSA (Supplementary Figure 1).

When grip strength of cut-off point for sarcopenia was $28 \mathrm{~kg}$ in men according to the latest recommendation by the Asian sarcopenia working group [20], the differences between sarcopenia group and non-sarcopenia group were listed at supplementary table 2 . The age $(\mathrm{OR}=1.131,95 \% \mathrm{CI}$ : 1.048-1.220; $P=0.001)$, gender $(\mathrm{OR}=0.124,95 \% \mathrm{CI}$ : $0.023-0.682 ; P=0.016)$ and CSA $(\mathrm{OR}=0.422,95 \% \mathrm{CI}$ : $0.207-0.861 ; P=0.018)$ were significant indicators associated with sarcopenia (Supplementary Table 3). AUC was 0.842 (95\% CI: 0.752-0.933, $\mathrm{P}<0.001)$ of the prediction equation consisting of age, gender and CSA (Supplementary Figure 2).

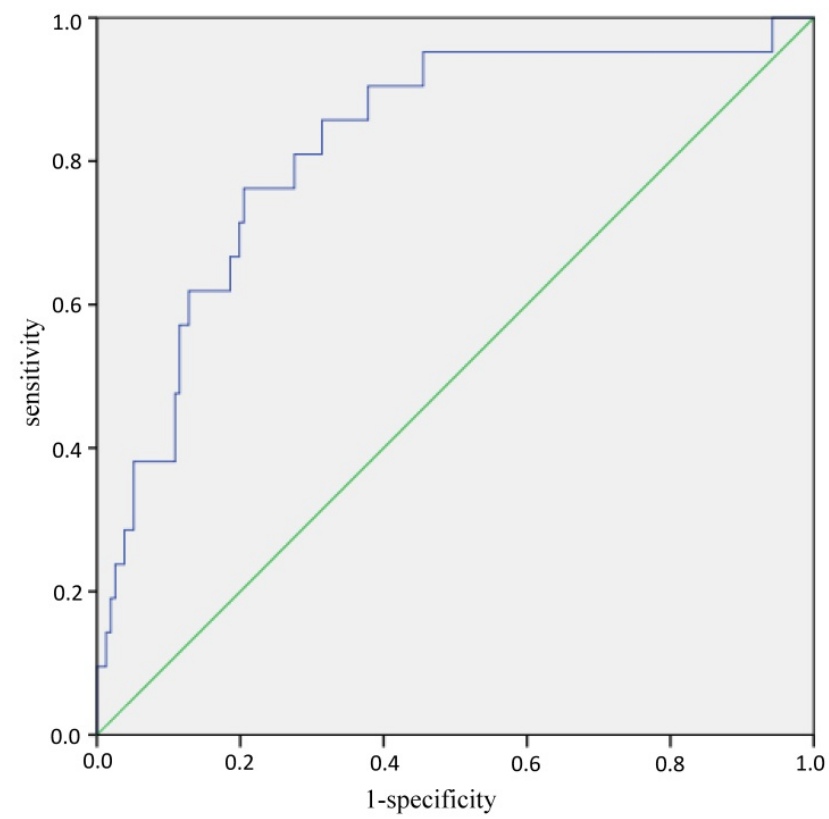

Figure 1. ROC curves of the equation in predicting sarcopenia. Note: The blue line is the tracing of ROC analysis of equation in predicting sarcopenia, the green line is the baseline. Abbreviations: ROC, receiver operating characteristic.

Table 4. Binary logistic regression analysis for sarcopenia

\begin{tabular}{llllll}
\hline variable & $\mathrm{B}$ & Wald & $\mathrm{P}$ & $\mathrm{OR}$ & $95 \% \mathrm{CI}$ \\
\hline Gender (women) & -0.908 & 0.991 & 0.320 & 0.403 & $0.067-2.411$ \\
Age(year) & $\mathbf{0 . 1 3 9}$ & $\mathbf{1 1 . 4 7 2}$ & $\mathbf{0 . 0 0 1}$ & $\mathbf{1 . 1 4 9}$ & $\mathbf{1 . 0 6 0 - 1 . 2 4 6}$ \\
BMI & -0.152 & 1.602 & 0.206 & 0.859 & $0.678-1.087$ \\
FT(mm) & -0.588 & 2.848 & 0.091 & 0.556 & $0.281-1.099$ \\
MT(mm) & 0.268 & 2.947 & 0.086 & 1.307 & $0.963-1.775$ \\
CSA(cm2) & $\mathbf{- 0 . 7 6 5}$ & $\mathbf{4 . 2 4 5}$ & $\mathbf{0 . 0 3 9}$ & $\mathbf{0 . 4 6 5}$ & $\mathbf{0 . 2 2 5 - 0 . 9 6 3}$
\end{tabular}

Notes: age, sex, BMI, MT, FT, and CSA were included in the binary logistic regression analysis of sarcopenia.

Abbreviations: BMI, body mass index; FT, fat thickness; mm, millimeter; MT, muscle thickness; CSA, cross-sectional area; cm, centimeter.

\section{Discussion}

The prevalence of sarcopenia was $1-29 \%$ for the elderly who live in the community and $14-33 \%$ in residents requiring long-term care according to the definition of European Working Group on Sarcopenia in Older People.[21] And the prevalence was reported to be $2.5-28.0 \%$ in men and $2.3-11.7 \%$ in women for the community-dwelling Japanese elderly.[22] In the study, the prevalence of sarcopenia was $16.3 \%$ and $10.8 \%$ respectively in men and women, which is accord with the previous reports. The high prevalence of sarcopenia in the elderly reminds us to pay more 
attention.

At present, the use of ultrasound to evaluate the muscle mass of the elderly has become a research hotspot. It's reported a good homogeneity of diameter and cross-sectional area of the rectus femoris measured by ultrasound and CT[23]. The similar results were confirmed by the contrast between ultrasound and MRI[24]. From these previous findings, the ultrasound could be proposed to be an effective measurement for assessing muscle mass. Here, we mainly assess the value of ultrasound measurement of the biceps brachii in sarcopenia.

Considering gender indicators, the study found the muscle mass and grip strength of men to be significantly greater than that of women, while the gait speed was not significantly different between the two groups. The MT and CSA of biceps brachii in man were significantly higher than those in women, while FT was significantly lower than those in women. This to some extent reflects the differences in muscle and adipose tissue distribution between men and women, and men seem to have more muscle tissue and less adipose tissue than women as previously stated[25].

Age is an independent risk factor for sarcopenia. The older the person gets, the greater the risk of sarcopenia. It has been reported that after 50 years of age, muscle mass decreases at a rate of about $1 \%$ to $2 \%$ per year, while muscle strength decreases at a rate of about $1.5 \%$ per year, and gradually accelerates to $3 \%$ per year after 60[3]. A prospective study in Asia also found that muscle mass and gait speed progressively decrease as humans get older[26]. In this study, binary logistic regression analysis showed that age was a significant factor associated with sarcopenia. Correlation analysis also revealed a negative correlation between age and grip strength or gait speed. Age and SMI also showed a significant negative correlation in elderly men, which is in line with the results of Japanese studies[27]. Moreover, the biceps brachii MT and CSA were negatively correlated with age.

Previous studies have shown a good correlation between ultrasound assessment of muscle and skeletal muscle mass measured by DXA[28-30]. Ismail et al[31] found a positive correlation between the muscle thickness measured by ultrasound and skeletal muscle mass measured by DXA. In addition to muscle mass, a strong correlation was observed between ultrasound parameters and muscle strength. [32]. The muscle echo strength had a significant positive correlation with grip strength [31]. Loss of muscle mass in specific parts of thighs assessed by ultrasound may be related to a decreased curve walking performance[33]. The muscle thickness of the forearm of the upper limb was also significantly correlated to the grip strength[34].In present study, positive correlations between CSA of the biceps brachii and SMI or grip strength were observed in both genders. But after controlling of age and BMI, the correlations between SMI and ultrasound parameters are not significant. These results indicated that the correlations between biceps ultrasound parameters and SMI or grip strength are associated with age and BMI.

The study by Kuyumcu et al[35] found the sarcopenia group defined by BIA and grip strength have significantly lower ultrasonic parameters of muscle thickness and fascicle length of gastrocnemius muscles than the non-sarcopenia group. Research also found that the thickness of the gastrocnemius muscle could effectively assess and predict low muscle mass[36]. Seymour et al[37] reported a positive correlation between the cross-sectional area of the rectus femoris measured by ultrasound and that by $\mathrm{CT}$, as well as the ultrasound parameters and the skeletal muscle mass measured by BIA. A strong positive correlation between the ulnar muscle thickness of the forearm measured by ultrasound and the skeletal muscle mass measured by DXA has been reported. [34, 38] All these data reflect the importance of ultrasound in sarcopenia. Our study found that biceps brachii CSA was significantly higher in the non-sarcopenia group than those in sarcopenia group. CSA as well as age was significant indicator for sarcopenia. Moreover, the equation of age, gender and CSA was a feasible method to predict the sarcopenia, which wasn't reported before.

\section{Limitation}

Although the study established the significance of biceps brachii CSA in the sarcopenia, the low number of participants and restricted region might have caused a certain degree of result bias. A larger data including multiple geographic regions and a range of health states is needed to identify clinically relevant thresholds for sarcopenia. The study just focuses on the biceps brachii, other sections of body isn't detected, which needs more studies to determine. The study mainly focuses on the value of ultrasound, the diet and exercise hadn't been investigated and the relationship between diseases or biochemical data and sarcopenia hadn't been explored.

\section{Abbreviations}

DXA: Dual-energy X-ray absorptiometry; BIA: bio-electrical impedance analysis; CT: computed tomography; MRI: magnetic resonance imaging; MT: muscle thickness; CSA: cross-sectional area; FT: fat thickness; ROC: receiver operating characteristic; AUC: Area Under Curve. 


\section{Supplementary Material}

Supplementary figures and tables.

http://www.medsci.org/v17p2947s1.pdf

\section{Acknowledgements}

This research was supported by National Key R\&D Program of China (2018YFC2000301, 2018YFC2001405).

\section{Competing Interests}

The authors have declared that no competing interest exists.

\section{References}

1. Rosenberg IH. Epidemiologic and methodologic problems in determining nutritional status of older persons. Proceedings of a conference. Albuquerque, New Mexico, October 19-21, 1988. The American journal of clinical nutrition. 1989; 50: 1121-235.

2. [Internet]. AIM Coalition Announces Establishment of ICD-10-CM Code for Sarcopenia by the Centers for Disease Control and Prevention. 2016. http://aginginmotion.org/news/2388-2/.

3. Rolland Y, Czerwinski S, Abellan Van Kan G, Morley JE, Cesari M, Onder $G$, et al. Sarcopenia: its assessment, etiology, pathogenesis, consequences and future perspectives. The journal of nutrition, health \& aging. 2008; 12: 433-50.

4. Chen L-K, Liu L-K, Woo J, Assantachai P, Auyeung T-W, Bahyah KS, et al. Sarcopenia in Asia: Consensus Report of the Asian Working Group for Sarcopenia. Journal of the American Medical Directors Association. 2014; 15: 95-101.

5. McLean RR, Kiel DP. Developing consensus criteria for sarcopenia: an update. Journal of bone and mineral research : the official journal of the American Society for Bone and Mineral Research. 2015; 30: 588-92.

6. Fielding RA, Vellas B, Evans WJ, Bhasin S, Morley JE, Newman AB, et al. Sarcopenia: an undiagnosed condition in older adults. Current consensus definition: prevalence, etiology, and consequences. International working group on sarcopenia. Journal of the American Medical Directors Association. 2011; 12: 249-56.

7. Cruz-Jentoft AJ, Baeyens JP, Bauer JM, Boirie Y, Cederholm T, Landi F, et al. Sarcopenia: European consensus on definition and diagnosis: Report of the European Working Group on Sarcopenia in Older People. Age and Ageing. 2010; 39: 412-23.

8. Heymsfield SB, Adamek M, Gonzalez MC, Jia G, Thomas DM. Assessing skeletal muscle mass: historical overview and state of the art. Journal of cachexia, sarcopenia and muscle. 2014; 5: 9-18.

9. Cruz-Jentoft AJ, Bahat G, Bauer J, Boirie $\mathrm{Y}$, Bruyère $\mathrm{O}$, Cederholm T, et al. Sarcopenia: revised European consensus on definition and diagnosis. Age and ageing. 2019; 48: 16-31.

10. Nishikawa H, Shiraki M, Hiramatsu A, Moriya K, Hino K, Nishiguchi S. Japan Society of Hepatology guidelines for sarcopenia in liver disease (1st edition): Recommendation from the working group for creation of sarcopenia assessment criteria. Hepatology Research. 2016; 46: 951-63.

11. Sergi G, De Rui M, Stubbs B, Veronese N, Manzato E. Measurement of lean body mass using bioelectrical impedance analysis: a consideration of the pros and cons. Aging clinical and experimental research. 2017; 29: 591-7.

12. Reiss J, Iglseder B, Kreutzer M, Weilbuchner I, Treschnitzer W, Kassmann $\mathrm{H}$, et al. Case finding for sarcopenia in geriatric inpatients: performance of bioimpedance analysis in comparison to dual X-ray absorptiometry. BMC geriatrics. 2016; 16: 52.

13. Nijholt W, Scafoglieri A, Jager-Wittenaar H, Hobbelen JSM, van der Schans CP. The reliability and validity of ultrasound to quantify muscles in older adults: a systematic review. Journal of cachexia, sarcopenia and muscle. 2017; 8: 702-12

14. Takai Y, Ohta M, Akagi R, Kato E, Wakahara T, Kawakami Y, et al. Applicability of ultrasound muscle thickness measurements for predicting fat-free mass in elderly population. The journal of nutrition, health \& aging. 2014; 18: 579-85.

15. Ticinesi A, Meschi T, Narici MV, Lauretani F, Maggio M. Muscle Ultrasound and Sarcopenia in Older Individuals: A Clinical Perspective. Journal of the American Medical Directors Association. 2017; 18: 290-300.
16. Scott JM, Martin DS, Ploutz-Snyder R, Matz T, Caine T, Downs M, et al. Panoramic ultrasound: a novel and valid tool for monitoring change in muscle mass. Journal of cachexia, sarcopenia and muscle. 2017; 8: 475-81.

17. Abe T, Thiebaud RS, Loenneke JP, Young KC. Prediction and validation of DXA-derived appendicular lean soft tissue mass by ultrasound in older adults. Age (Dordrecht, Netherlands). 2015; 37: 114.

18. Roberts HC, Denison HJ, Martin HJ, Patel HP, Syddall H, Cooper C, et al. A review of the measurement of grip strength in clinical and epidemiological studies: towards a standardised approach. Age and ageing. 2011; 40: 423-9.

19. Yang M, Hu X, Wang H, Zhang L, Hao Q, Dong B. Sarcopenia predicts readmission and mortality in elderly patients in acute care wards: a prospective study. Journal of cachexia, sarcopenia and muscle. 2017; 8: 251-8.

20. Chen L-K, Woo J, Assantachai P, Auyeung T-W, Chou M-Y, Iijima K, et al. Asian Working Group for Sarcopenia: 2019 Consensus Update on Sarcopenia Diagnosis and Treatment. Journal of the American Medical Directors Association. 2020; 21: 300-7.e2.

21. Cruz-Jentoft AJ, Landi F, Schneider SM, Zúñiga C, Arai H, Boirie Y, et al. Prevalence of and interventions for sarcopenia in ageing adults: a systematic review. Report of the International Sarcopenia Initiative (EWGSOP and IWGS). Age and ageing. 2014; 43: 748-59.

22. Kim $\mathrm{H}$, Hirano $\mathrm{H}$, Edahiro $\mathrm{A}$, Ohara $\mathrm{Y}$, Watanabe $\mathrm{Y}$, Kojima $\mathrm{N}$, et al. Sarcopenia: Prevalence and associated factors based on different suggested definitions in community-dwelling older adults. Geriatrics \& gerontology international. 2016; 16: 110-22.

23. Thomaes T, Thomis M, Onkelinx S, Coudyzer W, Cornelissen V, Vanhees L. Reliability and validity of the ultrasound technique to measure the rectus femoris muscle diameter in older CAD-patients. BMC medical imaging. 2012; 12: 7.

24. Sanada K, Kearns CF, Midorikawa T, Abe T. Prediction and validation of total and regional skeletal muscle mass by ultrasound in Japanese adults. European journal of applied physiology. 2006; 96: 24-31.

25. Janssen I, Heymsfield SB, Wang ZM, Ross R. Skeletal muscle mass and distribution in 468 men and women aged 18-88 yr. Journal of applied physiology (Bethesda, Md : 1985). 2000; 89: 81-8.

26. Auyeung TW, Lee SW, Leung J, Kwok T, Woo J. Age-associated decline of muscle mass, grip strength and gait speed: a 4-year longitudinal study of 3018 community-dwelling older Chinese. Geriatrics \& gerontology international. 2014; 14 Suppl 1: 76-84.

27. Shimokata H, Ando F, Yuki A, Otsuka R. Age-related changes in skeletal muscle mass among community-dwelling Japanese: a 12-year longitudinal study. Geriatrics \& gerontology international. 2014; 14 Suppl 1: 85-92.

28. Abe T, Thiebaud RS, Loenneke JP, Fujita E, Akamine T. DXA-Rectified Appendicular Lean Mass: Development of Ultrasound Prediction Models in Older Adults. The journal of nutrition, health \& aging. 2018; 22: 1080-5.

29. Berger J, Bunout D, Barrera G, de la Maza MP, Henriquez S, Leiva L, et al. Rectus femoris (RF) ultrasound for the assessment of muscle mass in older people. Archives of gerontology and geriatrics. 2015; 61: 33-8.

30. Abe T, Patterson KM, Stover CD, Geddam DA, Tribby AC, Lajza DG, et al. Site-specific thigh muscle loss as an independent phenomenon for age-related muscle loss in middle-aged and older men and women. Age (Dordrecht, Netherlands). 2014; 36: 9634.

31. Ismail C, Zabal J, Hernandez HJ, Woletz P, Manning H, Teixeira C, et al. Diagnostic ultrasound estimates of muscle mass and muscle quality discriminate between women with and without sarcopenia. Frontiers in physiology. 2015; 6: 302.

32. Watanabe Y, Yamada Y, Fukumoto Y, Ishihara T, Yokoyama K, Yoshida $\mathrm{T}$, et al. Echo intensity obtained from ultrasonography images reflecting muscle strength in elderly men. Clinical interventions in aging. 2013; 8: 993-8.

33. Abe T, Ogawa M, Loenneke JP, Thiebaud RS, Loftin M, Mitsukawa N. Relationship between site-specific loss of thigh muscle and gait performance in women: the HIREGASAKI study. Archives of gerontology and geriatrics. 2012; 55: e21-5.

34. Abe T, Counts BR, Barnett BE, Dankel SJ, Lee K, Loenneke JP. Associations between Handgrip Strength and Ultrasound-Measured Muscle Thickness of the Hand and Forearm in Young Men and Women. Ultrasound in medicine \& biology. 2015; 41: 2125-30.

35. Kuyumcu ME, Halil M, Kara O, Cuni B, Caglayan G, Guven S, et al. Ultrasonographic evaluation of the calf muscle mass and architecture in elderly patients with and without sarcopenia. Archives of gerontology and geriatrics. 2016; 65: 218-24.

36. Wang J, Hu Y, Tian G. Ultrasound measurements of gastrocnemius muscle thickness in older people with sarcopenia. Clinical interventions in aging. 2018; 13: 2193-9. 
37. Seymour JM, Ward K, Sidhu PS, Puthucheary Z, Steier J, Jolley CJ, et al. Ultrasound measurement of rectus femoris cross-sectional area and the relationship with quadriceps strength in COPD. Thorax. 2009; 64: 418-23.

38. Abe T, Fujita E, Thiebaud RS, Loenneke JP, Akamine T. Ultrasound-Derived Forearm Muscle Thickness Is a Powerful Predictor for Estimating DXA-Derived Appendicular Lean Mass in Japanese Older Adults. Ultrasound in medicine \& biology. 2016; 42: 2341-4. 